\title{
Chronic depression and anxiety in primary care: approaches to liaison
}

\author{
Keith Lloyd and Rachel Jenkins
}

Much attention has focused on prompt, accurate identification and treatment of depression and anxiety in primary care settings. Less is known about what to do when treatment is unsuccessful or the initial opportunity to intervene is missed. The long-term prognosis of depression and anxiety in primary care settings is not good: half of sufferers experience a relapsing remitting course and a quarter become chronic. There is also a relationship between chronicity and high service utilisation. The label "heartsink" has sometimes been applied to these patients, often pejoratively.

Initiatives such as the Defeat Depression Campaign (Paykel \& Priest, 1992) and Health of the Nation (Department of Health, 1994) are raising expectations and concerns about the morbidity to be addressed. It is important that strategies be developed within primary care to detect and manage these conditions so as not to divert secondary care resources from so-called severe mental illness. This paper looks at available practical strategies.

\section{What problems do these patients have?}

Between a third and a quarter of the workload of the average GP concerns mental health problems (Sharp \& Morrell, 1989). Depression and anxiety are the commonest mental illnesses seen in primary care while patients with psychosis constitute less than $10 \%$ of general practice psychiatry. A GP with a list size of 2500 identifies approximately 300 patients with a non-psychotic mental illness per year. This is termed 'conspicuous psychiatric morbidity'. Almost as many attenders again have an undiagnosed mental illness, most commonly depression or anxiety. This is the 'hidden psychiatric morbidity'. Only a small proportion of the mentally disordered who consult GPs reach the specialist psychiatric services. The majority are managed in primary care (Table 1) (Goldberg \& Huxley, 1992).

Defining chronicity as persistence of the index episode for more than two years, the greatest number of patients with chronic illnesses are encountered in primary care settings as are the greatest opportunities for prevention and treatment (Lloyd \& Jenkins, 1994). Depression and anxiety are among the commonest chronic conditions seen in primary care (Lyons et al, 1992; Scott, 1992). However, many primary care patients do not fit easily into a diagnosis of major depression or generalised anxiety disorder but have some symptoms of both. For practical purposes it is probably most helpful to think of depression and

Table 1. Goldberg \& Huxley's five levels and four filters on the pathways to psychiatric care

Setting

Period prevalence (n/1000 at risk/year)
Level 1: The community $260-315$
1st filter-----The decision to consult-
Level 2: Total primary care morbidity
230
2nd filter-----GP recognition-
Level 3: Conspicuous psychiatric morbidity 101.5
3rd filter--.-.-The decision to refer-
Level 4: Mental illness services
20.8
4th filter----Admission to psychiatric beds-
Level 5: Psychiatric in-patients
$3.8-6.7$

Keith Lloyd is Senior Lecturer in Mental Health at the University of Exeter. Dr Lloyd has extensive clinical and research experience in the primary care of mental illness.

Rachel Jenkins is Honorary Senior Lecturer at the Institute of Psychiatry, London. Dr Jenkins has carried out a variety of research studies of depression and anxiety in primary care and set up educational initiatives with primary care teams about depression. 
anxiety as dimensions rather than categories, although some disagree (Goldberg \& Huxley, 1992). Such patients may not reach the threshold for either disorder but show considerable morbidity and persistent symptoms (Johnstone \& Goldberg, 1976). Many experience symptoms of other physical and psychiatric disorders during their lifetimes.

Poorer short-term outcome is associated with initial severity of symptomatology, significant concomitant physical illness, severe social problems and material circumstances, genetic risk score and personality factors (Mann et al, 1981; Goldberg \& Huxley, 1992). Mortality is also increased among patients with conspicuous psychiatric morbidity referred on to psychiatric out-patients (Sims, 1973). According to US figures minor depression results in more days lost from work than major depression because of the former's greater prevalence. Only chronic heart disease produces more disability (Eisenberg, 1992). These patients are clearly not the "worried well".

\section{The importance of intervention}

There is a clear health gain for the patient from improved detection and management because detection improves prognosis and effective management improves the prognosis still further (Paykel \& Priest, 1992; Freemantle et al, 1993; Drummond, 1994). It is important to improve management in order to avoid the consequences

Box 1. General practitioner requirements from out-patient services

Rapid assessment

Shorter referral-appointment interval

Better communication

Clear management guidelines

Statement of objectives of treatment

Predicted response, complications and sideeffects

Six-monthly review plans for chronic patients

Clearly stated role of GP and specialist in treatment

Clarification of prescribing responsibilities Information booklets of therapies available

(After Strathdee, 1994) of chronicity such as considerable stress, and a low quality of life. Additionally there are considerable occupational problems such as sickness absence, labour turnover, problems with colleagues, poor performance and accidents. There can be problems for the children of chronically ill parents; they are more vulnerable to emotional and cognitive impairment, which in turn can predispose to adult mental illness on maturity, as well as having an adverse effect on the children's ultimate intellectual attainment. (Jenkins, 1992; Lloyd \& Jenkins, 1994). Besides persistent morbidity, a small proportion kill themselves. It has been argued that $90 \%$ of completed suicides had some form of mental disorder, most frequently depression; $66 \%$ had consulted their GP in the previous month; $40 \%$ had consulted their GP in the last week; and 33\% expressed clear suicidal ideation (Department of Health, 1994). This remains a controversial area.

Chronic depression, persistent unresolved grief states, chronic phobias, and tranquilliser dependency, may also place an excessive burden on health services. From a survey of women aged 20 to 45 years registered with two south London general practices, Corney \& Murray (1988) found that high attendance (defined as more than 10 consultations per year) was significantly associated with psychiatric morbidity, young age, lower socioeconomic group, concomitant physical symptoms, and receipt of a prescription for psychotropic medication within the last 12 months. High attenders were more likely to have sought help from other agencies in addition to their GP. Depressed patients with predominantly somatic presentations of depression are more likely than non-somatisers to use both medical and mental health services (Bass, 1994).

\section{Resources available to the GP and the primary care team}

Having emphasised the scale of the problem, the particular advantages of the GP's position, and the scope for preventive activity in general practice, how can busy GPs possibly deliver, in the midst of all the other competing obligations? They clearly need help and support, and plenty of it. How can this best be achieved?

Several developments in the past have arisen from this need, and some have been thoroughly evaluated. They have generally built on the practice of attaching secondary care personnel to the primary care team. So, for example, schemes of attached social workers have proven valuable 
in the treatment of people with chronic depression related to chronic social difficulties (Corney, 1984). Schemes of attached psychologists have been evaluated in the treatment of anxiety disorders, and in cognitive therapy of depression (Robson, 1992). Community Psychiatric Nurse attachments are now probably the most common form of attachment. A recent thorough evaluation by Gournay \& Brooking (1994) demonstrated the enormous opportunity cost entailed in withdrawing CPNs from the long-term severely mentally ill to treat depression and anxiety.

\section{A problem of numbers}

There is a problem with the attachment of secondary care personnel to the primary care setting in order to manage the patients for the GP because of the sheer numbers involved. There are approximately 30000 GPs in the country, each of whom will have 300-600 patients with depression and anxiety in any one year, and there are 2000 consultant general psychiatrists. Therefore each psychiatrist would need to have close links with 15 GPs, and could realistically only help each GP with a tiny proportion of all their depressed patients. Similarly, there is one CPN between five and ten GPs (the precise ratio varying across the country). If we take the best case of one CPN to every five GPs, we know that on average, each GP has seven patients with chronic severe mental illness (mostly chronic schizophrenia) who require supervision, support, family interventions and so on from a CPN (Kendrick et al, 1991). He or she then already has a caseload of 35 . It is easy to see that if each GP also refers three or four depressed patients, the CPN's working week has been overloaded (unless care is withdrawn from those with severe mental illness). Meanwhile, only a tiny dent is made on the GPs load of several hundred depressed patients. The severely mentally ill now, more than ever, need concentrated efforts in continuing care during and after they leave

Box 2. Models of primary care liaison

Informal meetings

Shifted out-patients

Consultation model

Consultation-liaison model hospital. CPNs will often be their case managers and/or key workers, and there are substantial concerns that this important work is being jeopardised by the shift of CPNs' caseloads from being primarily focused on the severely mentally ill to being focused on those with minor psychiatric morbidity (Gournay \& Brooking, 1994).

\section{The role for the psychiatrist}

Out-patient referral is the traditional route for obtaining a psychiatric opinion on a patient with chronic anxiety or depression. Strathdee (1994) surveyed 154 GPs in Camberwell to ask them what they wanted from out-patient services (Box 1). Many of the GPs concerns related to communication. At a simple level, the referral letter and reply can help improve communication (King \& Pullen, 1994). One approach is to use a standardised letter one side of A4 in length giving details of problems, management (physical, psychological, and social) and care programme.

The main limitation of the out-patient referral route is the small numbers of patients who can be managed in this way. As outlined above, a significant increase in the numbers of referrals dealt with via this route would seriously impair the secondary care team's ability to manage patients with psychotic mental illness. The alternative is to move into primary care.

Over the last 25 years more and more psychiatrists have established attachments to primary care settings and a variety of liaison models have emerged (Box 2). The "shifted out-patients" model, the most simple and common arrangement, was first described by Tyrer (1984). The psychiatrist conducts a usual out-patient clinic except that it takes place in a surgery. He can offer assessment, crisis intervention and 'hands on' management in the surgery. Patients often prefer being seen in a primary care setting and supervision of trainees is relatively easy. However, no research has been conducted to see if this method helps the primary heath care team improve their own management skills. A major problem is providing this kind of service to all the practices in a sector. According to the "consultation model" the psychiatrist advises the GP about management at regular intervals and also sees patients in primary care if required.

In the 'consultation-liaison' model the psychiatrist attends practice meetings to discuss management problems with the primary health care team. 
He or she may then see the patient accompanied by the GP or other member of the PHCT or practice attached CPN (Creed \& Marks, 1989). More patients can be discussed in this way and there is better GP/psychiatrist contact (Goldberg \& Huxley, 1992).

\section{A framework for liaison}

Having decided upon one of these approaches, the specific needs of the liaison setting need to be considered. A suggested framework for assessing referrals is given in Box 3 and explained in more detail below:

The referral: what for, why now, why worry? The psychiatrist may wish to think about: why is this person being brought to my attention now? What are the major concerns about this person? What is the relevant history? Is there a history of challenging behaviours or selfharm? How does this person respond to new professionals or changes in their management?

Diagnoses and personality: What is the likely psychiatric diagnosis/diagnoses? Does this person have comorbid disorders such as alcohol or substance abuse or tranquilliser dependency? What is the role of personality factors and coping style? It is important to emphasise that a diagnosis of personality disorder does not equal "untreatable" nor exclude other psychiatric diagnoses or physical and social problems. Tyrer (1994) proposes a simple classification of personality disorders seen in general practice into antisocial, withdrawn, dependent or inhibited and outlines simple management strategies.

Physical factors: Does this person have co-morbid physical illnesses? If so, how are they being treated and has the management of psychiatric problems been given an equal priority?

Somatisation: does this person have medically unexplained physical symptoms? Is the practice agreed on how far they should investigate those symptoms? Is it appropriate to educate the patient to re-attribute their symptoms?

Psychological factors: what is the role of life events and developmental factors? What are their coping strategies and cognitive style? Are there relevant family and couple issues which should be addressed? What do the family/ partner/carer understand about the person's condition? What psychological interventions has the person received from health social services or voluntary sector providers? What does the person stand to gain and lose by recovery?

Social factors: what are the sources of stress and support in this person's life? Consider particularly occupation relationships, marital/ cohabitation, family, social life, money, housing. What is the relevance of gender, ethnic and social class factors?

Level of functioning: How well does this person function in their activities of daily living? Do they need further support or might recovery mean losing some of their existing support?

Medication: has the patient had an appropriate course of an appropriate drug at adequate doses for long enough? How does the patient view the role of medication, what is compliance like and what side-effects are they experiencing? Are they receiving treatments that will exacerbate their psychiatric condition?

Organisation of care: who currently sees this person, how often and what for? How often do they consult and what happens in the consultation? Would regular booked appointments with a member of the PHCT help structure interaction? Is there a role for interview skills training? What patient literature does the practice have available? Does the practice have a directory of available health, social and voluntary sector agencies and what is locally available? How could communication about this patient be best improved?

In addition to the patient-specific help suggested above, it might also be helpful to think about the

Box 3. A framework for consultation-liaison

The referral: 'What for, why now, why worry, why change?'

Diagnoses and personality

Physical factors

Somatisation

Psychological factors

Social factors

Level of functioning

Medication

Organisation of care 
practice's strategy for identifying patients with chronic anxiety and depression. A number of simple screening instruments exist which can facilitate this process (Lloyd \& Jenkins, 1994). Computerised screening may have an increasing role to play in the future. High consulters can be identified from the practice computer and may merit screening for undiagnosed chronic depression and anxiety. High consulters known to the GPs may have under-treated depression and anxiety.

Above all, the main aim is to support the primary health care team (PHCT) in their management of difficult cases by reviewing what the issues are, what has been tried so far and what other possibilities there are. The intention is to offer assessment advice and support rather than active intervention in the majority of cases. This approach can be supplemented with teaching and educational materials for the PHCT.

\section{Educational materials for the PHCT and patient}

An alternative strategy, to shifting secondary care personnel into the primary care setting, is for secondary care personnel to teach the primary care teams to handle these patients themselves, i.e. to have a supportive and educational role rather than a direct 'hands on' role in relation to these patients with minor psychiatric morbidity.

A number of initiatives have been developed that can be adapted for local use or approached for advice. For example The Royal College of Psychiatrists has produced educational material for patients and professionals on depression and anxiety. Much self-help literature is available for the management of anxiety disorders (Drummond, 1994). A special primary care version is being developed of the 10th revision of the International Classification of Diseases (ICD-10 PHC) which presents simple diagnostic and management guidelines for common mental disorders such as depression and anxiety (Goldberg, 1994).

There is a national network of regional GP mental health fellows coordinated by Dr Andree Tylee. They are available as a resource for practices to develop their care of mental illness. Facilitators have been assisting primary care in developing their management of common chronic physical illnesses. A study has been undertaken to see if they can take on the same task of helping practices manage mental health (Lloyd \& Jenkins, 1995).
Gask and colleagues (Gask, 1992) in Manchester have developed a method to train GP trainers to teach communication skills using video and audio feedback techniques. Teaching packages have been developed to improve the detection and management of common mental disorders. These techniques are also available for experienced practitioners.

Psychological treatments developed in hospital settings are not always appropriate or available in primary care. Problem-solving therapy has been developed in Oxford as a simple, brief effective treatment that can be carried out by the PHCT. It has been shown to be an effective treatment for anxiety disorders of otherwise poor prognosis (Mynors et al, 1992).

\section{Making more help available}

The GP, meanwhile, needs more 'hands', and these can be obtained by strengthening the primary care team by the employment of practice nurses and counsellors and strategic deployment of health visitors and district nurses. The growth of counsellors in general practice is now well established, although we still need more careful evaluative studies of the effectiveness of the brief psychotherapies used by counsellors, and the indications for such treatment (Freemantle et al, 1993). Most GPs already employ practice nurses, health visitors and district nurses but these have traditionally only been used for physical and not psychological problems. There are a number of studies under way which can be brought to the GP's attention as practical strategies for improving the care of chronic mental illnesses in primary care.

A study is currently taking place at the Institute of Psychiatry to examine whether practice nurses can identify those patients at risk of chronicity. The University of Exeter is developing and evaluating a generalisable package to audit the quality of mental health care for depression. Oldfield Park Surgery, Bath, is funded to create a 'model' general practice for the purposes of optimal care of patients with psychological illness and related emotional problems consulting in that practice. The Royal College of Psychiatrists' and the Royal College of General Practitioners' 5 year 'Defeat Depression' campaign comprises both professional and public education initiatives. The aims are to increase knowledge among health care professionals about recognition and treatment of depressive illness and to enhance public awareness of depressive disorders. 
Sometimes it is not possible to sort the problem out locally. The Maudsley Hospital has a specialist tertiary referral service led by Professor Anthony Mann for general practice patients with chronic depression and anxiety. The key to the service is that it is extremely flexible and tailored to the individual patient. For some patients an afternoon spent at the GP's surgery with staff from the centre might be sufficient to enable them to continue being treated at primary care level. Others might require a longer assessment during which the aim is to listen, measure, watch and gain trust, not to reassure or treat.

All the above projects can be contacted for more details.

\section{Conclusions}

The best treatment of chronic anxiety and depression is prevention (Lloyd \& Jenkins, 1994). Simple strategies exist to improve the detection and management of depression and anxiety which are among the most common chronic disorders encountered in primary care settings. Yet they are less likely to be practice priorities than other common disorders such as asthma, diabetes, coronary heart disease and cancer. Much ignorance and stigma are attached to depression and anxiety. Sufferers are sometimes unwilling to seek help. People may feel embarrassed to mention their problems. They may feel there is not enough time or that the doctor will be unsympathetic. Doctors are only too aware of the importance of not missing physical illnesses but depression and anxiety are often overlooked, leading to chronicity and increased morbidity. This may be due to time constraints, the belief that nothing can be done, or the notion that they are understandable and therefore do not require treatment. The psychiatrist has a key role to play in getting the balance right.

\section{References}

Bass, C. (1994) Somatisation. In Prevention in Psychiatry (eds E. Paykel \& R. Jenkins), pp 188-197. London: Gaskell.

Corney, R. (1984) The effectiveness of attached social workers in the management of depressed female patients in general practice. Psychological Medicine, 14 (Monograph Suppl. 6.), 1-47.

- \& Murray, J. (1988) The characteristics of high and low attenders at two general practices. Social Psychiatry \& Psychiatric Epidemiology, 23, 39-49.

Creed, F. \& Marks, B. (1989) Liaison psychiatry in general practice: a comparison of the liaison attachment scheme and the shifted out-patient clinic models. Journal of the Royal College of General Practitioners, 39, 514-517.

Department of Health (1994) Health of the Nation Key Area Handbook of Mental Illness (2nd edn), pp 19-24. London: HMSO.

Drummond, L.M. (1994) Anxiety. In Psychiatry and General Practice Today (eds I. Pullen, G. Wilkinson, A. Wright, et al), pp 112-134. London: Gaskell.

Eisenberg, L. (1992) Treating depression and anxiety in primary care: closing the gap between knowledge and practice. New England Journal of Medicine, 326, 1080-1084.

Freemantle, N., Long, N., Mason, J., et al (1993) The treatment of depression in primary care. Effectiveness Health Care Bulletin, 5, 2-12.

Gask, L. (1992) Teaching psychiatric interview skills to general practitioners. In Prevention of Depression and Anxiety - the Role of the Primary Care Team (eds R. Jenkins, J. Newton \& J. Young), pp 39-45. London: HMSO.

Goldberg, D. \& Huxley, P. (1992) Common Mental Disorders. London: Routledge.

- (1994) Epidemiology of mental disorder in general practice. In Psychiatry and General Practice Today (eds I. Pullen, G. Wilkinson, A. Wright, et al), pp 36-44. London: Gaskell.

Gournay, K. \& Brooking, J. (1994) Community psychiatric nurses in primary health care. British Journal of Psychiatry, 165, 231238.

Jenkins, R. (1992) Depression and anxiety: an overview of preventive strategies. In Prevention of Depression and Anxiety - the Role of the Primary Care Team (eds R. Jenkins, J. Newton, \& R. Young), pp 11-21. London: HMSO.

Johnstone, A. \& Goldberg, D. (1976) Psychiatric screening in general practice: a controlled trial. Lancet, $\mathbf{i}, 605-608$.

Kendrick, T., Sibbald, P., Burns, T., et al (1991) Role of general practitioners in care of long-term mentally ill. British Medical Journal, 302, 508-510.

King, M. \& Pullen, I. (1994) Communication between general practitioners and psychiatrists. In Psychiatry and General Practice Today (eds I. Pullen, G. Wilkinson, A. Wright, et al), pp 251-264. London: Gaskell.

Lloyd, K. \& Jenkins, R. (1994) Primary Care. In Prevention in Psychiatry (eds E. Paykel \& R. Jenkins), pp 198-209. London: Gaskell.

- \& - (1995) The economics of depression in primary care: Department of Health Initiatives. British Journal of Psychiatry, 166 (Suppl. 27), 60-62.

Lyons, R.A., Caroll, D., Doherty, K., et al (1992) General practice estimates of the prevalence of common chronic conditions. Irish Medical Journal, 85, 22-24.

Mann, A. H., Jenkins, R., \& Belsey, E. (1981) The 12 month outcome of patients with neurotic disorder in general practice. Psychological Medicine, 11, 535-550.

Mynors-Wallis, L.M. \& Gath, D.H. (1992) Brief psychological treatments. International Review of Psychiatry, 4, 301-306.

Paykel, E. S. \& Priest, R. G. (1992) Recognition and management of depression and general practice: consensus statement. British Medical Journal, 305, 1198-1202.

Robson, M. (1992) Counselling in general practice: options for action. A clinical psychology. In Prevention of Depression and Anxiety - the Role of the Primary Care Team (eds R. Jenkins, J. Newton \& R. Young), pp 77-81. London: HMSO.

Scott, J., Eccleston, D., \& Boys, R. (1992) Can we predict the persistence of depression? British Journal of Psychiatry, 161, 633-637.

Sharp, D., \& Morrell, D. (1989) The psychiatry of general practice. In Scientific Approaches in Epidemiological Psychiatry. Essays in Honour of Michael Shepherd (eds P. Williams, G. Wilkinson \& K. Rawnsley), pp 404-419. London: Routledge.

Sims, A. (1973) Mortality and neurosis. Lancet, ii, 1072-1075.

Strathdee, G. (1994) Psychiatry and general practice - a psychiatric perspective. In Psychiatry and General Practice Today (eds I. Pullen, G. Wilkinson, A. Wright, et al), pp 22-35. London: Gaskell.

Tyrer, P. (1984) Psychiatric clinics in general practice: an extension of community care. British Journal of Psychiatry, 145, 9-14. 
(1994) Personality disorder. In Psychiatry and General Practice Today (eds I. Pullen, G. Wilkinson, A. Wright, et al), pp 180193. London: Gaskell.

\section{Multiple choice questions.}

1 Primary care attenders with chronic depression and anxiety

a consume less primary care time than psychosis

b are more likely to be referred to secondary care than managed in primary care

c are largely the "worried well"

d have a better outcome if their disorder is detected and treated

e show a raised Standardised Mortality Ratio

2 Among completed suicides

a $45 \%$ had some form of mental disorder

b the most common mental disorder was depression

c $66 \%$ have consulted their GP in the last month

d $20 \%$ have consulted their GP in the last week

e $33 \%$ expressed clear suicidal ideation

3 The following statements are true regarding the organisation of care:

a There is approximately one general psychiatrist to every $15 \mathrm{GPs}$

b practice linked CPNs should be encouraged to focus on chronic depression and anxiety as their core activity

c The shifted out-patient model improves the management skills of the primary health care team

d the consultation liaison model is one of the most efficient way of giving consultant input to primary care

e patients prefer being seen in primary care rather than out-patient settings

4 The following have been demonstrated to be effective treatments for depression and/or anxiety:

a tricyclic antidepressants

b problem solving therapy

c counselling

d cognitive-behavioural therapy

e selective serotonin re-uptake inhibitors

\begin{tabular}{|ccclll||}
\hline \multicolumn{7}{|c|}{ MCQ answers } & & & & \\
1 & 2 & 3 & 4 & \\
a F & a F & a $T$ & a T \\
b F & b T & b F & b T \\
c F & c T & c F & c F \\
d T & d F & d T & d T \\
e T & e T & e T & e T \\
\hline
\end{tabular}

